\title{
Selbstverständnis und Rolle von Personalberatungen bei der Besetzung von Führungspositionen
}

\section{Prof. Dr. Carsten Wippermann}

Professor für Soziologie, KSFH Benediktbeuern, Leiter des DELTAInstituts für Sozial- und Ökologieforschung $\mathrm{GmbH}$, Penzberg

„Mehr Frauen in Führungspositionen!“ - dieses von der Mehrheit der Bevölkerung unterstützte Vorhaben steht in Deutschland weit oben auf der Agenda der Gleichstellungspolitik und zunehmend auch der Wirtschaftspolitik. Besondere Verantwortung kommt neben den Entscheidern in den Unternehmen den Personalberatungen zu, die eine Schlüsselfunktion bei der Stellenbesetzung haben. Denn sie werden eingeschaltet, um in einem Beratungsprozess die optimale Besetzung offener Stellen in Bezug auf Kandidatinnen und Kandidaten innerhalb und außerhalb des Unternehmens zu reflektieren. Im Jahr 2010 waren in Deutschland etwa 1.900 Beratungsunternehmen tätig, fast 45.000 Beratungsmandate wurden bearbeitet.

Das war 2010 bis 2012 Anlass für das Bundesministerium für Familie, Senioren, Frauen und Jugend (BMFSFJ), im Rahmen einer sozialwissenschaftlichen Untersuchung zu klären, welche Funktion und Wirkung Personalberater/innen im Prozess der Besetzung von Führungspositionen haben und welche Haltung sie selbst in Bezug auf das Ziel „Mehr Frauen in Führungspositionen!“ einnehmen.

Aus dem breiten Spektrum an Kompetenzen von Personalberatungen sind vor allem zwei zu betonen: Zum einen haben Personalberater/innen möglichst aktuelle Informationen über grundsätzlich in Frage kommende Personen mit ihrem beruflichen Werdegang, ihren Fähigkeiten und Erfolgen, auch zu ihren persönlichen Präferenzen und Hintergründen. Dazu arbeiten Personalberater/innen oft mit Unterstützung von Datenbanken, die sie als Pool nutzen, pflegen und erweitern, aber auch durch die Pflege persönlicher Netzwerke, zum Beispiel mit Personen, die sie bereits vermittelt haben. Diese individuelle (analoge und digitale) „Datenbank“ ist eine wichtige Quelle und wird fortlaufend gepflegt. Daneben verfügen sie über umfangreiche Kenntnisse von Märkten, Branchen, Vergütungsstrukturen und Karrierewegen. Zum anderen sind Personalberater/innen Meister der diskreten Gesprächsführung zur sukzessiven Auslotung der jeweiligen Interessen und Ziele sowie der bilateralen Passung zueinander. Sie müssen sowohl die fachlichen Kompetenzen differenziert aufnehmen und vergleichen können, als auch menschliche und soziokulturelle Aspekte. Insofern lernen sie mögliche Kandidatinnen und Kandidaten von verschiedenen Seiten kennen, erfahren durch Gespräche und Beobachtungen vieles über Einstellungen, Motive, Ziele, Habitus, Mentalitätsmuster und weitere Momente dessen, was später nicht auf offiziellen Papieren erscheint. Insofern sind Personalberater/innen eine gute Auskunftei zu den Fragen:

- Warum gibt es heute immer noch so wenige Frauen in Führungspositionen? Was sind Ursachen und Motive?
- Was sind typische Verhaltensmuster von Frauen und Männern im Prozess der Besetzung einer vakanten Führungsposition?

- Welche Kompetenzen (Skills) sind in Führungspositionen erforderlich?

- Was sind geeignete und wirksame Instrumente, damit mehr Frauen in Führungspositionen kommen?

$\mathrm{Zu}$ diesen Fragen wurden im Rahmen einer qualitativen sozialwissenschaftlichen Untersuchung Personalberater/innen interviewt. Sie kamen aus großen, mittleren und kleinen Personalberatungsunternehmen; deutscher und internationaler Provenienz (Hauptsitz in USA, Großbritannien, Frankreich). Das Spektrum reichte auch von relativ jungen Personalberatungen bis hin zu den langjährig Etablierten. Methodisch ist die Untersuchung so angelegt, dass die qualitative Repräsentanz der Marktakteure abbildet. In diskreten Expertengesprächen wurden zur Hälfte Frauen und Männer mit Projektverantwortung und Key Account für bestimmte Branchen und Top-Kundschaft befragt.

Bereits in den ersten Minuten des Interviews thematisierten fast alle Personalberater/innen von sich aus das Thema „Frauenquote“. Die politisch diskutierte gesetzliche Quotenregelung sowie die von einigen Unternehmen bereits beschlossenen betrieblichen Ziele für einen Mindestanteil von Frauen in Führungspositionen sind bei Personalberatungen derzeit ein zentrales Thema, denn es betrifft ihr Alltagsgeschäft unmittelbar. Daher haben Personalberater/innen eine dezidierte, klare Haltung zur gesetzlichen Quotenregelung. Das Spektrum der Erfahrungen sowie der Einstellungen der Personalberater/ innen ist insgesamt sehr breit. Gleichwohl zeigen sich in Bezug auf ihr Verhältnis zur Förderung von Frauenkarrieren zwei Hauptgruppen:

- Typ I: Ein Teil der Personalberater/innen lehnt eine Frauenquote für Führungspositionen vehement ab. Eine gesetzliche Quote werde dem geringen Marktpotenzial an Frauen nicht gerecht und gefährde die notwendige Qualität bei der Stellenbesetzung. Diese Haltung haben vor allem auf dem deutschen Markt tätige internationale Personalberatungen (aus USA, GB) sowie etablierte deutsche Personalberatungsfirmen, deren wichtigste Kunden große (z.B. börsennotierte) Unternehmen sind.

- Typ II: Ein anderer Teil der Personalberater/innen diagnostiziert im Markt hingegen ein weitgehend unerschlossenes Potenzial an qualifizierten Frauen. Den Frauenanteil über eine gesetzliche Quote sowie flankierende Maßnahmen zu erhöhen, sei für Unternehmen, Frauen und auch den Markt der Personalberatungsbranche eine Chance und längst überfällig. Diese Haltung haben vorwiegend relativ junge, kleinere und mittelgroße Personalberatungen.

Interessant ist, dass beide ihre Haltung mit ihrer exzellenten Marktkenntnis begründen, die in der Diagnose über das Potenzial jedoch völlig gegensätzlich ausfällt. In ihrer Grundo- 
rientierung grenzen sich Personalberaterinnen/Personalberater des einen Typs in rationaler Argumentation mit Verweis auf ihre exzellente Kenntnis des Marktes und des Machbaren von Personalberaterinnen/Personalberatern des anderen Typs kategorisch und harsch ab.

\section{Typ I: Konservativ-etablierte Personalberater/innen: Im Effekt „Gatekeeper“}

Personalberater/innen des Typs I legen ausdrücklich Wert auf die Feststellung, es gebe bei der Besetzung von Führungspositionen heute keine Geschlechterunterschiede mehr, sondern es komme allein auf die fachliche und vor allem persönliche Eignung an. Notwendige Kompetenzen für Führungsaufgaben seien vor allem die Fähigkeit zur Bewältigung komplexer Sachlagen, Kommunikationsfähigkeit, Empathie, intuitive Fähigkeiten, mittel- und langfristiges Denken, die Bereitschaft, neue Wege zu gehen mit Kreativität und Pioniergeist sowie spezifische Kriterien, die im gemeinsam erarbeiteten Stellenprofil formuliert sind. Das Geschlecht als Selektionskriterium spiele keine Rolle, es komme allein auf die Qualität der individuellen Person an.

Personalberater/innen, so die Argumentation, seien professionelle Dienstleister und suchten daher eine qualitativ optimale, aber auch effiziente und zügige Lösung im Sinne ihrer Klienten. Um im Wettbewerb bestehen zu können, müssten sie ihren Markt sehr gut kennen, sowohl hinsichtlich des quantitativen Potentials für typische Hierarchiestufen und Profile, als auch bezüglich der qualitativen (fachlichen, internationalen, politischen, kommunikativen, repräsentativen, kulturellen ...) Anforderungen und Güte. Und hier betonen Personalberater/innen dieses ersten Typus, aufgrund ihrer Marktkenntnis „absolut gegen jede gesetzliche Quote“ zu sein, die einen Mindestanteil von Frauen in Führungspositionen vorschreibt. Sie begründen dies vor allem mit drei Argumenten:

- Mangelnde „Masse“: Für Top-Führungspositionen gebe es zu wenige geeignete Frauen mit entsprechenden Voraussetzungen, es fehle derzeit noch die Masse, das Potenzial für die Auswahl sei zu gering. Für oberste Führungsaufgaben Aufsichtsrat; Vorstand; Direktorium, Bereichsleitung von Konzernen, Geschäftsleitung mittelständischer Unternehmen - seien mehrere Jahre Berufserfahrung eine unbedingte Voraussetzung. Wer nicht schon Berufs- und Branchenerfahrung (auch in Leitungsfunktionen) von mindestens 15 oder 20 Jahren habe, bringe die erforderliche Qualität nicht mit. Das erfordere eine jahrzehntelange Aufbauentwicklung im Markt, denn bis vor wenigen Jahren wären die meisten Frauen noch nicht durchgängig berufstätig gewesen und hätten nicht die erforderliche Berufsausbildung. Dazu komme, dass viele Berufszweige (vor allem naturwissenschaftliche und technologische) bis heute das Image als typische Männerdomäne hätten. Das habe dazu geführt, dass es in den meisten Branchen auch heute noch zu wenige Frauen gibt, die diese MINT-Fächer studiert hätten (dieses bessere sich allmählich, so dass langsam mehr Frauen in das mittlere Management aufsteigen würden), und kaum
Frauen mit der notwendigen langjährigen Berufserfahrung. Eine gesetzliche Frauenquote, die dies ignoriere, provoziere für die Unternehmen unausweichlich das Risiko minderer Qualität bei der zwanghaften Besetzung von Führungspositionen mit Frauen.

- Mangelnde Bereitschaft: Von den wenigen möglicherweise geeigneten Frauen würden die meisten gar nicht in TopFührungspositionen wollen - so die Aussage der Personalberater/innen vom Typ I. Diese Frauen hätten die notwendige Ausbildung und vielleicht auch das Potenzial, aber sie würden andere Prioritäten setzen. Wichtiger als ein Sprung nach oben auf der Karriereleiter sei ihnen die Vereinbarkeit von Familie und Beruf, die Balance von Leben und Arbeiten. Sie würden sich nicht ganz und gar dem Beruf verschreiben können und wollen, nicht rund um die Uhr verfügbar sein, nicht nachts auch noch zu Terminen müssen, nicht ständig auf Reisen sein (europaweit, zwischen den Kontinenten). Sie blieben lieber im mittleren Management und seien zufrieden mit dem, was sie hätten. Und das müsse man respektieren!

- Mangelnde Zielstrebigkeit, zu frühe Beförderung: Viele akademisch und fachlich geeignete Frauen seien in ihrer Berufslaufbahn und vor allem in ihrer Persönlichkeitsentwicklung noch nicht reif für eine Führungsfunktion, würden aber dazu gedrängt. Sie zeigten sich in den Vorstellungsgesprächen unsicher, damit nicht professionell, nicht machtbewusst, nicht stark genug, und scheitern im Selektionsverfahren daher mitunter kläglich. Sie würden vor dem entscheidenden Auswahlgremium nicht bestehen, „weil sie vor der dort sitzenden Männerrunde zusammenklappen und versagen". Sie seien zwar fachlich höchst kompetent, wirkten ansonsten auch souverän und kommunikativ, doch in der entscheidenden Situation würden sie (noch) nicht bestehen. Der Karrieresprung käme für sie zu früh, sie müssten noch einige Jahre Erfahrung sammeln.

Diese Personalberaterinnen und Personalberater sehen (nur) die Frauen selbst gefordert, ihre Einstellungen und Verhandlungsmuster zu ändern, und keinen wesentlichen Handlungsoder Änderungsbedarf bei sich selbst oder den Unternehmen. Sie demonstrieren im Interview allerdings große Zuversicht, dass durch die selbstbewussteren jüngeren Generationen in den nächsten zehn bis $20 \mathrm{Jahren/in} \mathrm{den} \mathrm{nächsten} \mathrm{ein} \mathrm{bis} \mathrm{zwei}$ Generationen der Anteil von Frauen in TOP-Positionen automatisch steigen werde. Hinsichtlich des Ziels „Mehr Frauen in Führungspositionen “ übernehmen diese Personalberater/ innen die Rolle der „Gatekeeper“, die im Effekt dazu beitragen, dass Frauen der Zugang zu Führungspositionen weiter verwehrt bleibt und nur in Ausnahmefällen möglich ist. Insofern sind diese Personalberater/innen Teil im Abwehrriegel des „Gläserne-Decke“-Mechanismus.

\section{Typ II: Progressive Personalberater/innen: „Türöffnende“}

Davon abweichende Erfahrungen und Einstellungen haben vor allem jüngere, kleinere oder auf spezifische Unternehmensarten spezialisierte Personalberatungen sowie jene, die 
das Ziel „Mehr Frauen in Führungspositionen!“ als längst überfällig erachten. Sie beobachten, dass sich der Markt verändert habe: Immer mehr Frauen seien hoch qualifiziert für Top-Managementfunktionen und hätten die entsprechende Voraussetzung, Motivation und Bereitschaft, diese Verantwortung zu übernehmen.

Aufgrund der Internationalisierung der Märkte sowie der in Deutschland seit Jahren kritisch geführten Debatte über den geringen Anteil von Frauen in Führungspositionen sehen diese Personalberater/innen die Unternehmen in Deutschland zunehmend unter Druck. Ein Teil der Unternehmensleitungen wolle bei vakanten Stellen weiter nur einen Mann. Zunehmend mehr Unternehmen und auch innovative Personalberater/innen sähen aber eine dringende Notwendigkeit für eine strukturelle Abkehr von den gewohnten Routinen bei der Besetzung von Führungspositionen, weil männerhomogene Führungsmannschaften heute nicht mehr die optimale Voraussetzung für nachhaltigen Unternehmenserfolg böten. Sie würden als unzeitgemäß angesehen, da sie das Image des Unternehmens beschädigten. Vielfalt in Führungsetagen sei sozial gerecht und ökonomisch notwendig, um angesichts einer immer komplexeren Umwelt gut aufgestellt zu sein und um Chancen und Risiken zu erkennen. Diese Personalberater/innen sehen ihre Aufgabe und Kompetenz darin, die Unternehmen bei der Besetzung von neuen Führungspositionen auch in diesem ganzheitlichen Zusammenhang zu beraten. Ökonomische Rationalität und Geschlechtergerechtigkeit begründen und befördern sich wechselseitig. Die Unternehmenslandschaft sei in der Führung dann gut aufgestellt, wenn sie ein Spiegelbild der Gesellschaft sei und gerade in Führungspositionen den Erfordernissen einer lebensereignisorientierten Balance von Beruf und Familie gerecht werde.

$\mathrm{Zu}$ behaupten, es gäbe die notwendige Menge an geeigneten Frauen nicht, halten Personalberater/innen dieses Typs für eine Ausrede. Von jenen Gatekeeper-Personalberatungen, die an ihren bewährten Routinen festhalten wollen („die Großen, Alten, Konservativen und Platzhirsche im Markt"), grenzen sich diese Personalberatungen ab. Sie stimmen allerdings in einer Beobachtung mit ersteren überein: Beim Erstkontakt und im weiteren Bewerbungsprozess wollen qualifizierte Frauen meist ganz sicher sein, dass sie der Aufgabe gewachsen sind, sie trauen sich oft nicht, wagen den Sprung nicht. Wenige rufen selbstbewusst „Klar, das mache ich!“. Frauen würden in der Mehrheit die 100-Prozent-Gewissheit wollen, der Aufgabe gewachsen zu sein, bevor sie diese in Angriff nehmen. Und Frauen seien deutlich weniger mutig und bereit, für sich selbst Werbung zu machen, für sich zu trommeln. Das unterscheide sie von Männern, die im eigenen Interesse offensiver seien. Aber anders als Personalberater/innen des ersten Typs ziehen diese Personalberater/innen des zweiten Typs nicht den Schluss, es sei allein an den Frauen, ihre Einstellung und ihr Verhalten zu verändern. Auch die Unternehmen sowie die Personalberater/innen seien gefordert, neue Wege zu gehen zur Sicherung der Zukunftsfähigkeit der Unternehmen und aufgrund ihrer gesellschaftlichen Verantwortung. Dafür müsse man die vorhandenen Frauen strategisch in den Blick nehmen, Rechercheroutinen innovativ verändern und diesen Frauen eine Chance geben - ohne dies als Test zu begreifen. Denn Frauen könnten ebenso gut wie Männer einen Konzern, ein Unternehmen und Mitarbeiter/innen führen. Mehr Frauen für Führungspositionen zu rekrutieren, hätte vermutlich in einigen Bereichen sogar den Effekt, dass die Qualität der Führungsfunktionen in den Unternehmen steige, weil dann die traditionelle informelle Männerquote aufgebrochen wäre und es wirklich um Leistung ginge.

Dass der Anteil von Frauen in Führungspositionen seit Jahren sehr gering ist trotz hoher beruflicher Qualifikation, liegt nach Erfahrung dieser Personalberater/innen an drei ungünstigen Beziehungsmustern:

- Insbesondere in männerdominierten Branchen wie Maschinen-/Anlagenbau, Metallindustrie, Energie, Automobil, Schienenindustrie u.a. stünden an der Spitze der Unternehmen fast ausschließlich Männer im Alter von etwas über 50 bis Mitte 60 Jahren. Diese lebten selbst privat in klaren traditionellen Rollenmustern: Die Gattin sei sozial engagiert und halte den Rücken frei. Berufstätige Frauen kennen Männer in oberster Führungsposition - so die Erfahrung dieser Personalberater/innen - primär als Assistentin und Mitarbeiterin im mittleren Management. „Es fällt diesen Herren schwer sich vorzustellen, dass eine Frau die fachliche und persönliche Qualifikation für eine höhere Führungsfunktion hat und sich durchsetzt." Das Frauenbild dieser Männer, die über die vakante Führungsposition entscheiden und dem beauftragten Personalberatungsunternehmen im Briefing-Gespräch das gewünschte Positionsprofil kommunizieren, sei trotz der aus Gründen der political correctness demonstrierten Wertschätzung gegenüber Frauen von Vorurteilen und Stereotypen geprägt. Personalberater/innen bekämen im Aufnahmegespräch informell (niemals schriftlich) diese Ansichten und Präferenzen diskret mitgeteilt. Und die meisten Personalberater/ innen verstünden sich als verlängerter Arm der Unternehmen; jene Gatekeeper seien bemüht, die direkten, die indirekt formulierten oder auch nur vermuteten Vorstellungen umfassend zu erfüllen.

- Es gebe eine enge strategische Verbindung von Unternehmensleitung und Personalberatung. Diese werden im konkreten Fall jene Personalberatung beauftragen, die ihre Ziele, Interessen und Präferenzen kenne (meist aus jahrelanger $\mathrm{Zu}$ sammenarbeit) und erfülle und bei der das Risiko minimiert sei, dass bei einer Fehlbesetzung sie selbst dafür verantwortlich gemacht würden. Insofern würden renommierte große Personalberatungsunternehmen die an sie gestellten bzw. die von ihnen vermuteten Erwartungen bedienen. Eine Frau zu vermitteln, erscheine „konservativ-etablierten Personalberatungen" (Typ I: Gatekeeper) immer noch eher ein Risiko. Dabei sei im Markt zu beobachten, dass gerade diese „Platzhirsche" sich in der Außendarstellung und PR das Thema „Frauen“ auf die Fahne schreiben - es dann aber nicht wirklich praktizieren, sondern auf das mangelnde Angebot an 
geeigneten Frauen hinweisen und dazu auf ihre unüberholbare Marktexpertise verweisen. Das sei eine simple Projektion der Ursachen auf „die Frauen“ bzw. „den Markt“ mit dem Zweck, das eigene unveränderte Verhalten zu rechtfertigen. Dies gelte vor allem für Unternehmensberatungen aus dem anglo-amerikanischen Raum mit der Maxime des schnellen Projektabschlusses (und damit Effektivitäts- und Gewinnsteigerung für das Beratungsunternehmen), die am Ende „Gatekeeper“ statt „Türöffnende“ für Frauen in Führungspositionen seien.

- Es gebe in einigen Fällen eine enge strategische Verbindung von Personalberaterinnen/Personalberatern mit Top-Kandidaten: „Gute Personalberater halten mit ihren besten Pferden, die ein überaus hohes Jahreseinkommen haben, dauerhaft guten Kontakt". Wenn diese einen Vertrag unterschreiben, ist auch das daran bemessene Honorar der Personalberater/innen entsprechend hoch. Denn auch in Führungspositionen hätten Männer ein deutlich höheres Entgelt als Frauen, und insofern sei es im eigenen Interesse ökonomisch rational, einen Mann zu vermitteln und keine Frau. Darüber hinaus gebe es einige Fälle, bei denen vermittelte Kandidaten nach zwei, drei oder vier Jahren erneut wechseln wollen, woran die Personalberatung und der Top-Kandidat verdienten. Zu diesen strategischen Wechselkandidaten würden Personalberater/innen einen engen Kontakt halten. Diese „schwarzen Bücher" seien eine Ursache dafür, dass in Top-Führungspositionen immer wieder dieselben Namen auftauchten und es für Frauen schwer sei, für Personalberater/innen mit diesem strategischen Pool-Pflege-Konzept zusätzlich von ökonomischem Interesse zu sein.

Um für eine Veränderung der Verhältnisse und für mehr Frauen in Führungspositionen zu sorgen, halten Personalberater/ innen des Typs II daher eine gesetzliche Quotenregelung für den notwendigen und längst überfälligen Hebel. Eine gesetzliche Quotenregelung würde auch dafür sorgen, dass Unternehmen in absehbarer Zeit wieder Spiegel der gesellschaftlichen Verhältnisse bezüglich des Anteils von qualifizierten Frauen und Männern wären. Das müsse dann unbedingt auch zur Folge haben, dass in Führungspositionen die Frage der Vereinbarkeit von Beruf und Familie stärker in den Blick genommen werde und auch gezielt Mütter mit noch kleinen Kindern die Chance auf Führungsaufgaben bekommen können, wenn diese Frauen das wollen - das verändere Rollenbilder, Arbeitszeiten und Arbeitsroutinen. Die gesetzliche Quote wird von diesen Personalberater/innen an sich nicht geliebt, erscheint ihnen aber notwendig als Schub, um in einen anderen Gleichgewichtszustand zu kommen. Ohne dieses von außen gesetzte Rahmenkriterium werden sich die bestehenden Verhältnisse nur marginal und viel zu langsam ändern.

Personalberater/innen dieses Typs II haben Erklärungen dafür, warum andere (des Typs I) sich vehement gegen eine gesetzliche Quotenregelung aussprechen: Eine Quotenregelung sei mit sehr viel mehr Arbeit bei der Personalsuche verbunden, da die bestehenden Adresspools nicht ausreichend sind. Es sei einfacher, für die erste Führungsebene einen Mann zu finden als eine Frau. Eine Quote koste somit Zeit und Geld, erzeuge neuen Wettbewerb und sei damit ein Risiko für etablierte Personalberatungen mit ihren Bestandskunden.

Fazit: Personalberater/innen des Typs II stehen unter demselben Zeitdruck wie jene des Typs I, für eine vakante Führungsposition möglichst schnell („am besten vorgestern“) geeignete Kandidatinnen und Kandidaten für die Vorstellungspräsentation beim Unternehmen zu finden. Aber sie reflektieren die überkommenen Denkmuster und Suchroutinen kritisch: Vor dem Hintergrund ökonomischer Rationalität und gesellschaftlicher Vernunft halten sie es für zwingend geboten, dass in Unternehmen mehr Frauen in Führungspositionen sind. Sie zeigen nicht den Reflex, aufgrund des leichteren Findens fachlich qualifizierter Männer mit deren offensiverer Selbstpräsentation darauf zu schließen, dass es noch zu wenige geeignete und bereite Frauen im Markt gibt. Personalberater/innen des Typs II haben in verschiedenen Branchen die Erfahrung gemacht, dass es viele fachlich und persönlich qualifizierte Frauen für das Top-Management gibt und heute schon der Anteil von Frauen in Führungspositionen sehr viel höher sein könnte. Sie sind auch überzeugt, dass das quantitative Potenzial von Frauen sehr viel größer ist als gemeinhin angenommen, aber noch weitgehend unerschlossen, dass aber auch ein Teil der fachlich und persönlich qualifizierten Frauen noch lernen muss, sich aktiver und offensiver um diese Stellen zu bewerben.

Postscriptum: Die Ergebnisse dieser Studie wurden bei verschiedenen Gelegenheiten der Fachöffentlichkeit präsentiert. Spannend zu beobachten ist in anschließenden Diskussionen, mit welcher Schärfe die anwesenden Personalberater vom Typ I „Gatekeeper“ die Richtigkeit der zuvor beschriebenen Befunde bezweifeln. Dabei bestätigen diese Personalberater performativ genau das, was sie zurückweisen. Im Habitus demonstrieren sie ihre Überlegenheit und unantastbare Marktund Sachkenntnis. Absolut nicht hinnehmbar ist für sie offenbar, dass sie selbst Gegenstand einer differenzierten sozialwissenschaftlichen Untersuchung werden und ihre Einstellungen und ihr Verhalten nicht mehr im Lichte sakrosankter Überlegenheit und Rationalität erscheint, sondern relativiert wird im Horizont eigener ökonomischer Interessen. 